\title{
Discrete Time Crystals: Rigidity, Criticality, and Realizations
}

\author{
N. Y. Yao, ${ }^{1}$ A. C. Potter, ${ }^{1,2}$ I.-D. Potirniche, ${ }^{1}$ and A. Vishwanath ${ }^{1,3}$ \\ ${ }^{1}$ Department of Physics, University of California Berkeley, Berkeley, California 94720, USA \\ ${ }^{2}$ Department of Physics, University of Texas at Austin, Austin, Texas 78712, USA \\ ${ }^{3}$ Department of Physics, Harvard University, Cambridge, Massachusetts 02138, USA
}

(Received 5 November 2016; published 18 January 2017)

\begin{abstract}
Despite being forbidden in equilibrium, spontaneous breaking of time translation symmetry can occur in periodically driven, Floquet systems with discrete time-translation symmetry. The period of the resulting discrete time crystal is quantized to an integer multiple of the drive period, arising from a combination of collective synchronization and many body localization. Here, we consider a simple model for a onedimensional discrete time crystal which explicitly reveals the rigidity of the emergent oscillations as the drive is varied. We numerically map out its phase diagram and compute the properties of the dynamical phase transition where the time crystal melts into a trivial Floquet insulator. Moreover, we demonstrate that the model can be realized with current experimental technologies and propose a blueprint based upon a one dimensional chain of trapped ions. Using experimental parameters (featuring long-range interactions), we identify the phase boundaries of the ion-time-crystal and propose a measurable signature of the symmetry breaking phase transition.
\end{abstract}

DOI: 10.1103/PhysRevLett.118.030401

Spontaneous symmetry breaking — where a quantum state breaks an underlying symmetry of its parent Hamiltonianrepresents a unifying concept in modern physics [1,2]. Its ubiquity spans from condensed matter and atomic physics to high energy particle physics; indeed, examples of the phenomenon abound in nature: superconductors, Bose-Einstein condensates, (anti)ferromagnets, any crystal, and Higgs mass generation for fundamental particles. This diversity seems to suggest that almost any symmetry can be broken.

Spurred by this notion, and the analogy to spatial crystals, Wilczek proposed the intriguing concept of a "time crystal"a state which spontaneously breaks continuous time translation symmetry [3-5]. Subsequent work developed more precise definitions of such time translation symmetry breaking (TTSB) [6-8] and ultimately, led to a proof of the "absence of (equilibrium) quantum time crystals" [9]. However, this proof leaves the door open to TTSB in $[10,11]$ has demonstrated that quantum systems subject to periodic driving can indeed exhibit discrete TTSB [10-13]; such systems develop persistent macroscopic oscillations at an integer multiple of the driving period, manifesting in a subharmonic response for physical observables.

An important constraint on symmetry breaking in manybody Floquet systems is the need for disorder and localization [10-17]. In the translation-invariant setting, Floquet eigenstates are short-range correlated and resemble infinite temperature states which cannot exhibit symmetry breaking $[15,18,19]$. Under certain conditions, however, prethermal time-crystal-like dynamics can persist for long times [20,21] even in the absence of localization before ultimately being destroyed by thermalization $[17,22]$.
In this Letter, we present three main results. First, by exploring the interplay between entanglement, many body localization and TTSB, we produce a phase diagram for a discrete time crystal (DTC) [24]. The DTC, like other
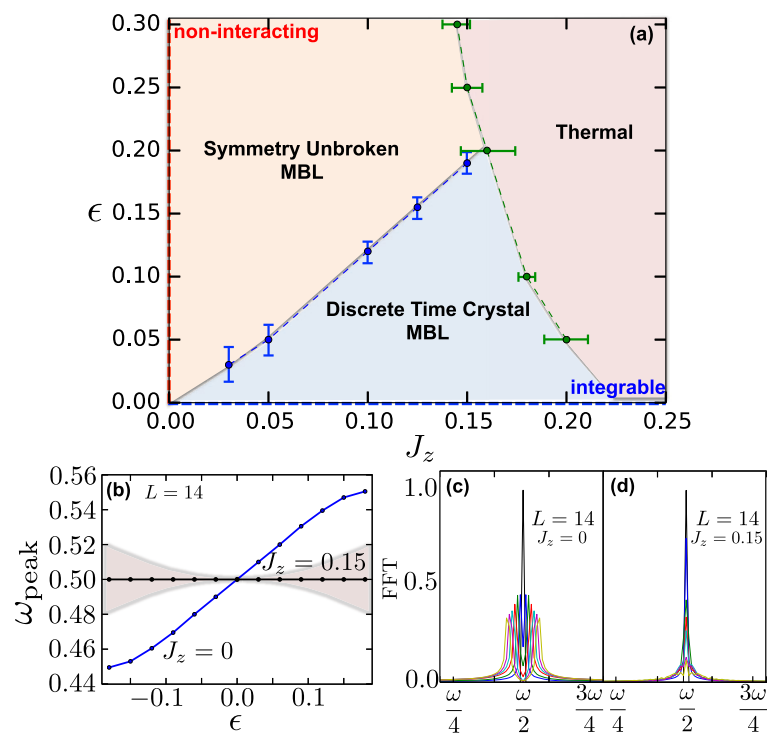

FIG. 1. (a) Phase diagram of the discrete time crystal as a function of interaction strength $J_{z}$ and pulse imperfections $\epsilon$. (b) Depicts the location of the subharmonic Fourier peak as a function of $\epsilon$. In the noninteracting case $\left(J_{z}=0\right)$, the peak tracks $\epsilon$, while in the interacting case $\left(J_{z}=0.15\right)$, the peak remains rigidly locked at $\omega / 2$. The pink region indicates the FWHM of the base of the $\omega / 2$ peak. Data are obtained at $L=14$ with $10^{2}$ disorder averages. (c)-(d) Representative realizations of the subharmonic Fourier response corresponding to $\epsilon$ in (b). All Fourier transforms are computed using $10<n<150$ Floquet periods. 
symmetry breaking phases, possess macroscopic rigidity and remains locked in its "collective" period, displaying a characteristic "plateau" at the location of its subharmonic Fourier response (Fig. 1). This is in stark contrast to free spins, which simply follow the period dictated by the driving. Second, we compute the scaling properties of the dynamical quantum critical point associated with the onset of TTSB, or equivalently, the quantum melting of the time crystal. Third, we propose an experimental realization of the DTC in a one-dimensional chain of trapped ions. Using experimental parameters, we identify the phase boundaries of the DTC and propose a measurable signature of the symmetry breaking phase transition.

Discrete time crystal.-Let us begin by considering a one dimension spin- $1 / 2$ chain governed by the binary stroboscopic Floquet Hamiltonian (with period $T=T_{1}+T_{2}$ ),

$H_{f}(t)= \begin{cases}H_{1} \equiv(g-\epsilon) \sum_{i} \sigma_{i}^{x}, & 0<t<T_{1} \\ H_{2} \equiv \sum_{i} J_{z} \sigma_{i}^{z} \sigma_{i+1}^{z}+B_{i}^{z} \sigma_{i}^{z}, & T_{1}<t<T\end{cases}$

where $\vec{\sigma}$ are Pauli operators and $B_{i}^{z} \in[0, W]$ is a random longitudinal field. To simplify the notation, we choose to work in units of $T_{1}=T_{2}=1$, where the Floquet evolution reduces to: $U_{f}=U_{2} U_{1} \equiv e^{-i H_{2}} e^{-i H_{1}}$. Throughout the remainder of the Letter, we work with $g=\pi / 2$ and note that for generic $\epsilon \neq 0$, the model does not exhibit any microscopic symmetries [13].

To gain some intuition for the nature of TTSB in this model, let us begin with the ideal decoupled limit where $\epsilon=J_{z}=0$. In the parlance of NMR, this simple case corresponds to a chain of decoupled spins undergoing "spin-echo" time evolution. To see this, let us consider a random initial product state, $|\psi\rangle=|\uparrow \downarrow \downarrow \uparrow \downarrow \cdots\rangle$, aligned along the $\hat{z}$ direction. The spin-echo unitary, $U_{1}=$ $e^{-i \pi / 2 \sum_{i} \sigma_{i}^{r}}$, flips each spin about the $\hat{x}$ axis, resulting in the oppositely polarized state, $\left|\psi_{1}\right\rangle=|\downarrow \uparrow \uparrow \downarrow \uparrow \cdots\rangle$. The second unitary results in only a global phase, $\phi$, as each spin is already aligned along $\hat{z},\left|\psi_{2}\right\rangle=e^{i \phi}|\downarrow \uparrow \uparrow \downarrow \uparrow \cdots\rangle$. Since each spin is flipped once per Floquet period, measuring a simple autocorrelation function, $R(t)=\left\langle\sigma_{i}^{z}(t) \sigma_{i}^{z}(0)\right\rangle$, at stroboscopic times (e.g. $T, 2 T, \cdots)$ yields a perfect train of oscillations [25].

These oscillations imply that $R(t)$ is $2 T$ periodic, a fact best captured by its subharmonic Fourier response at $\omega / 2$ - half the drive frequency [Fig. 1(c)]. This seems to fit the picture of TTSB and raises the question: are decoupled spins undergoing "spin echo" a discrete time crystal? The answer lies in the lack of stability to perturbations $[21,26,27]$. In this decoupled limit, any imperfection in the spin-echo pulse (e.g., $\epsilon \neq 0$ ) immediately destroys the $\omega / 2$ subharmonic. In particular, for $\epsilon>0$, the unitary, $U_{1}=e^{-i(\pi / 2-\epsilon) \sum_{i} \sigma_{i}{ }^{x}}$, leads to beating in $R(t)$ and a splitting of the $\omega / 2$ Fourier peak [Fig. 1(c)].
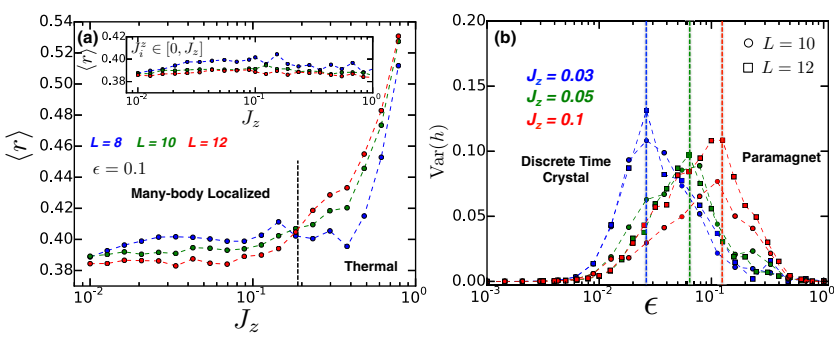

FIG. 2. (a) Level statistics ratio for $\epsilon=0.1$ as a function of $J_{z}$. For $L=8$ and $L=10$, we perform $\sim 10^{4}$ disorder realizations and for $L=12$, we perform $\sim 10^{3}$ disorder realizations. There is a clear crossing at $J_{z} \approx 0.18$, indicating the transition. Additional weak disorder in the interactions, $J_{i}^{z} \in\left[0, J_{z}\right]$, preserves localization over the same parameter range (inset). (b) Variance of the $\omega / 2$ Fourier peak magnitude as a function of $\epsilon$. We observe a clear peak at the transition, which exhibits a nearly linear scaling with increasing $J_{z}$.

Turning on sufficiently strong Ising interaction $\left(J_{z}>0\right)$ leads to a qualitatively different story $[10-13,20]$. For perfect echo pulses $(\epsilon=0)$, the autocorrelation function looks identical to the decoupled case, exhibiting the same normalized Fourier peak at $\omega / 2$ [Fig. 1(d)]. Crucially, imperfections $(\epsilon>0)$ no longer lead to a splitting of the $\omega / 2$ Fourier peak, demonstrating the robustness of the system's subharmonic response [Fig. 1(d)]. Herein lies the essence of the discrete time crystal-despite imperfect spin rotations, collective synchronization from the interactions maintains robust oscillations at half the driving frequency. This rigidity is evinced in Fig. 1(b) [28], where the location of the normalized Fourier peak is plotted as a function of $\epsilon$; for finite interactions, this peak is locked at precisely $\omega / 2$.

To explore the phase diagram of the discrete time crystal, we perform extensive numerical simulations to probe both the localization and symmetry breaking phase transitions [29]. As the DTC is only stable in the presence of localization, we begin by characterizing the MBL transition via the quasienergy level statistics ratio, $\langle r\rangle=\min \left(\delta_{n}, \delta_{n+1}\right) /$ $\max \left(\delta_{n}, \delta_{n+1}\right)$, where $\delta_{n}=\mathcal{E}_{n+1}-\mathcal{E}_{n}$ is the $n$th quasienergy gap [10,30]. Upon averaging $\langle r\rangle$ over both disorder and the quasi-energy spectrum, one expects the value in the thermal phase to approach the circular orthogonal ensemble limit of 0.527 , while the value in the localize phase should approach the Poisson limit of 0.386. Figure 2(a) depicts $\langle r\rangle$ as a function of $J_{z}$ for $\epsilon=0.1$, where one observes a clear transition at $J_{z} \approx 0.18$. The evolution of this thermalization transition point for general $J_{z}$ and $\epsilon$ is shown in Fig. 1(a) (green line). Interestingly, the transition exhibits a weak flow toward larger $J_{z}$ at small $\epsilon$, consistent with the integrability of $\epsilon=0$ line.

That the thermalization transition occurs for such weak interactions (more than an order of magnitude smaller than the disorder width) is somewhat surprising; a simple explanation may be that the (imperfect) spin-echo unitary, which flips each spin by approximately $180^{\circ}$, is nearly 
canceling the random field between the two pieces of the binary drive, leading to effectively weaker disorder. This is consistent with our observation that turning on additional disorder $\left(J_{i}^{z} \in\left[0, J_{z}\right]\right)$ in the Ising interactions, which are invariant under a uniform spin rotation, leads to a significantly enhanced region of localization [Fig. 2(a), inset].

Let us now turn to diagnosing the TTSB transition, which enables us to establish the existence of the discrete time crystal phase and locate its phase boundaries. We will use a combination of four signatures (at infinite temperature): 1) magnitude, 2) variance, and 3) exponential (in system size) persistence - of the subharmonic Fourier peak, and 4) mutual information between distant sites [11]. We note that a number of other probes of the DTC phase have also been proposed, including certain eigenstate correlations and responses [13].

We have already encountered the first signature while probing the rigidity of the $\omega / 2$ subharmonic response. As one increases the strength of the drive imperfections, $\epsilon$, the magnitude, $h$, of the $\omega / 2$ peak decreases [Fig. 1(d)] and eventually, becomes completely washed out when one transitions into the trivial paramagnet [25]. The second signature originates from strong critical fluctuations in $h$ near the TTSB transition. This results in a sharp peak in the variance of $h$ and enables one to quantitatively locate the transition in moderate system sizes. As shown in Fig. 2(b), increasing $J_{z}$ strengthens the rigidity of the DTC, shifting the melting transition toward larger detuning, $\epsilon$. We identify the third signature by computing the finite time scale where the $\omega / 2$ Fourier peak drops below amplitude 0.05 ; in the DTC phase, this time scale increases exponentially in system size, while in the trivial phase, it exhibits a significantly weaker dependence [11-13]. The final signature (Fig. 3) relates to the long-range mutual information [11] and will be discussed below in the context of the critical scaling properties of the TTSB transition. As illustrated in Fig. 1(a), the combination of these four diagnostics allows us to establish the TTSB transition point as a function of $J_{z}$ and $\epsilon$ (blue line).

Quantum melting transition.-Having mapped out the phase diagram of the DTC, we turn to an analysis of the critical properties of the TTSB transition [25,31]. We obtain the universal scaling properties of this dynamical quantum phase transition by mapping the Floquet evolution to a "hidden" effective static Ising model whose excited state critical properties can be exactly obtained by renormalization group methods [34-38]. Though the TTSB transition falls into the random Ising universality class, we will see that the hidden character of the Ising model introduces notable differences in physical scaling properties.

For simplicity, our analytic analysis will be performed in a model where the TTSB transition is tuned via transverse fields instead of spin-echo imperfections (e.g., $\epsilon=0$ ) [11-13]. While the conclusions will be identical, this approach allows us to compute the effect of $U_{1}$ exactly and
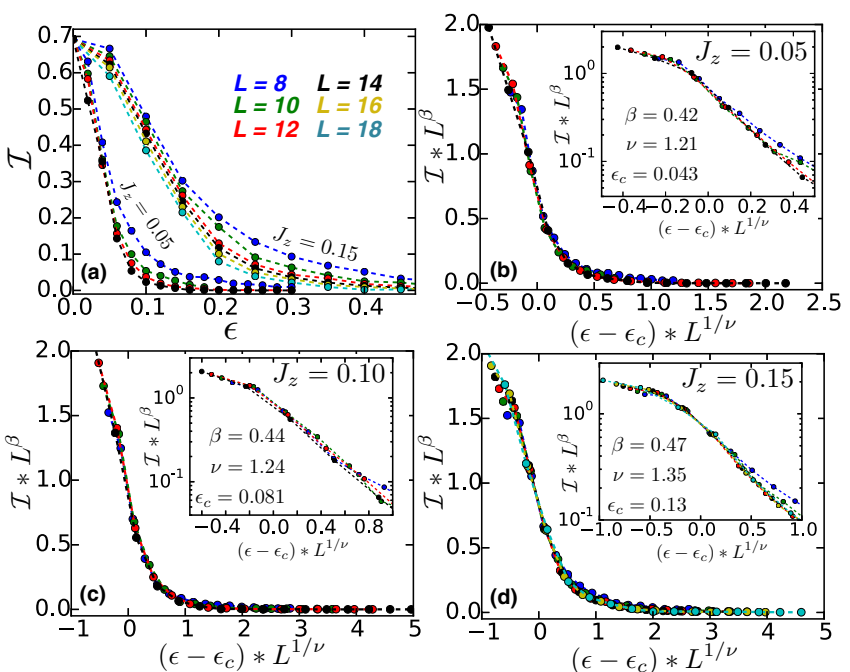

FIG. 3. (a) Finite size flow of the mutual information between spins on opposite ends of a length $L$ chain. For small detuning, $\epsilon \approx 0$, there is nearly full $\mathcal{I}=\log 2$, long range mutual information, which drops dramatically upon leaving the TTSB phase for large $\epsilon$. (b)-(d) Scaling collapse of $\mathcal{I}$ to the functional form $\left(1 / L^{\beta}\right) f\left(L /\left|\epsilon-\epsilon_{c}\right|^{-\nu}\right)$ optimized over the parameters $\beta$ and $\nu$. Insets depict the collapsed data with a semilogarithmic $y$ axis. Averaging over all $J_{z}$ yields numerical estimates for the critical exponents: $\beta_{\mathrm{ED}} \approx 0.4 \pm 0.1$ and $\nu_{\mathrm{ED}} \approx 1.3 \pm 0.1$; we note that the error bar associated with these exponents ignores the ambiguity in the location of the transition.

then to treat $U_{2}$ in a high frequency expansion for $J_{z} T$, $|\vec{B}| T \ll 1$. To this end, we consider a modified $H_{2} \rightarrow H_{2}^{\prime}=$ $\sum_{i} J_{z} \sigma_{i}^{z} \sigma_{i+1}^{z}+\sum_{\alpha=x, y, z} B_{i}^{\alpha} \sigma_{i}^{\alpha}$, where $B^{x}$ controls the transition and $B^{y}$ is added to avoid unintentional microscopic symmetries [13].

This model exhibits a hidden emergent Ising symmetry $\tilde{S}$ [13], and is in fact, related by a finite depth unitary transformation, $U_{\mathrm{FD}}$, to a driven transverse field Ising model with $B^{y, z}=0$. In particular, $U_{\mathrm{FD}} U(T) U_{\mathrm{FD}}^{\dagger}=e^{-i H_{\mathrm{TFM}} T} \prod_{i} \sigma_{x}^{i}$, where $H_{\mathrm{TFIM}}=\sum_{i} \tilde{J}_{i}^{z} \sigma_{i}^{z} \sigma_{i+1}^{z}+\tilde{B}_{i}^{x} \sigma_{i}^{x}$ has a conventional (e.g., on site and Hamiltonian independent) symmetry $S=\prod_{i} \sigma_{i}^{x}$, and $\tilde{J}_{i}^{z}, \tilde{B}_{i}^{x}$ are spatially random quantities, given the disordered character of $U_{\mathrm{FD}}$. Recast in slightly different terms, the original Floquet evolution has an emergent "hidden" Ising symmetry (which is quasi-local and Hamiltonian specific) generated by $\tilde{S}=U_{\mathrm{FD}}^{\dagger}\left(\prod_{i} \sigma_{x}^{i}\right) U_{\mathrm{FD}}$ [12]. In the supplementary information, we provide a general scheme to explicitly construct this emergent symmetry in a way that reveals its physical connection to time-translation symmetry.

To probe the nature of the TTSB transition, our strategy is to consider time evolution for two Floquet periods, $U(2 T)=$ $U_{\mathrm{FD}}^{\dagger} e^{-2 i H_{\mathrm{TFIM}} T} U_{\mathrm{FD}}$. Crucially, unlike $U(T)$, this unitary takes the form of evolution under a local transverse field Ising Hamiltonian. Since long-time evolution can always be decomposed into repeated evolutions by $U(2 T)$ followed by partial evolution for up to a single period, the late-time 
properties of the system are governed by those of the excited eigenstates of $H_{\text {TFIM }}$. For strong disorder, these states exhibit a nonergodic quantum phase transition between a trivial MBL phase and an Ising symmetry breaking magnetic glass phase. This transition falls into the same universality class as the zero temperature random Ising transition [34]. Thus, the $Z_{2}$ discrete time-crystal melting transition, at strong disorder, falls into the universality class of a "hidden" random Ising transition [34-37].

A few remarks are in order. The key difference between this "hidden" Ising transition and the conventional transition is the following: The scaling fields, $\Sigma^{\alpha}$ of this "hidden" Ising transition, i.e., those that exhibit $\overline{\left\langle\Sigma^{\alpha}(r) \Sigma^{\beta}(0)\right\rangle}=\delta_{\alpha \beta} / r^{2 \Delta_{\alpha}}$ (where the overbar indicates disorder averaging), are related to those of $H_{\mathrm{TFIM}}$ by $U_{\mathrm{FD}}$. Because of the absence of any microscopic symmetries in the underlying DTC Hamiltonian, the original spins will generically have overlap with all scaling fields: $\sigma_{i}^{\mu}=\sum_{i, j, \alpha} c_{i j, \alpha}^{\mu} \Sigma_{j}^{\alpha}(\mu=x, y, z)$, where $c_{i j, \alpha}^{\mu}$ are nonuniversal coefficients that depend on the microscopic details of the lattice and decay exponentially in $|i-j|$. Thus, generic spin-spin correlation functions will also pick up contributions from all scaling fields: $\overline{\left\langle\sigma_{i}^{\mu} \sigma_{j}^{\nu}\right\rangle}=$ $\sum_{i^{\prime} j^{\prime} \alpha} c_{i i^{\prime}, \alpha}^{(\mu)} c_{j j^{\prime}, \alpha}^{(\nu)}\left(1 /\left|r_{i^{\prime} j^{\prime}}\right|^{2 \Delta_{\alpha}}\right) \approx\left(1 /|i-j|^{2 \Delta_{\alpha_{*}}}\right)$. For large separations, the decay of these correlation functions will be dominated by the scaling field, $\alpha_{*}$, with the slowest decay (i.e., minimal scaling dimension, $\Delta_{\alpha_{*}}$ ). In the case of the 1D random Ising transition, the magnetization has the slowest decay $\sim 1 / r^{\beta}$, where $\beta=2-\varphi$ and $\varphi=(1+\sqrt{5}) / 2$ is the golden ratio [34].

Because of the strong randomness character of the transition, there is a marked difference between the mean scaling behavior just discussed and the typical scaling behavior. Indeed, the $1 / r^{2-\varphi}$ power-law behavior of all local mean correlation functions results from rare regions that are unusually large, well-ordered, and dominate the average [34]. Typical correlation functions, on the other hand, all decay significantly faster than any power law, namely, as a stretched exponential: $\left\langle\Sigma^{\alpha}(r) \Sigma^{\beta}(0)\right\rangle_{\text {typ }} \sim$ $\delta_{\alpha \beta} e^{-\sqrt{r}}$. Similarly, the typical and mean scaling properties will also be governed by two different diverging length scales: $\xi_{\text {typ }} \sim\left|\epsilon-\epsilon_{c}\right|^{-\nu_{\text {typ }}}$ and $\xi_{\text {avg }} \sim\left|\epsilon-\epsilon_{c}\right|^{-\nu_{\text {avg }}}$, with correlation length exponents, $\nu_{\text {typ }}=1$ and $\nu_{\text {avg }}=2$.

While the above discussion focuses on critical eigenstate properties, in an experiment, one is interested in manifestations of criticality in dynamical signatures. To this end, one can examine the critical temporal decay of the aforementioned $\omega / 2$ Fourier peak. A sharp definition of this mixed time or frequency object can be obtained through the Wigner distribution function: $C^{a b}\left(\omega_{0}, t\right) \equiv \int_{0}^{\infty} d \tau e^{-i \omega_{0} \tau}\left\langle\sigma^{a}(t+\tau) \sigma^{b}(\tau)\right\rangle$, which, due to the "hidden" Ising structure of the transition will decay asymptotically as the slowest decaying scaling field [35]

$$
C^{a b}\left(\omega_{0}=\frac{\omega}{2}, t\right) \sim \frac{1}{\log ^{2-\varphi} t} .
$$

This logarithmically slow decay contrasts with both the power-law decay characteristic of trivial MBL phases, $C_{\mathrm{MBL}}^{a b} \sim t^{-p}$ and the exponential decay characteristic of a thermalizing system, $C_{\text {thermal }}^{a b} \sim e^{-t}[39]$.

Critical scaling of mutual information.-Having elucidated the scaling structure of the TTSB transition, we now perform a numerical exploration of the time-crystal-melting transition for the original model [Eq. (1)]. In particular, we compute the mutual information, $\mathcal{I}(L)$, between the first and last site of the spin chain as a function of $\epsilon$ for fixed $J_{z}$ (Fig. 3) [11]. As depicted in Fig. 3(a), the mutual information exhibits a clear finite size flow, sharpening with increasing system size. To explore the critical properties of the transition, we conduct a finite size scaling analysis of this data, based on our analytic understanding of the transition. In analogy to the disordered Ising transition, the TTSB critical point can be viewed as having a broad distribution of nearly ordered time-crystal clusters. The mutual information between two spins separated by $L$ is of order unity when they belong to the same cluster and exponentially small otherwise. Hence, at criticality, $\mathcal{I}(L)$ tracks the probability for two spins to be in the same cluster, which scales as $\sim L^{-\beta}$ [34]. For $\epsilon \approx \epsilon_{c}$ near the transition, the mutual information will then follow the universal scaling form: $\mathcal{I} \sim\left(1 / L^{\beta}\right) f(L / \xi)$, where $\xi \sim\left|\epsilon-\epsilon_{c}\right|^{-\nu}$ is the correlation length of the incipient time-crystal order.

In Figs. 3(b)-3(d), we perform a two parameter scaling collapse on the numerical data for $\mathcal{I}$, by plotting $L^{\beta} \mathcal{I}(L)$ versus $\left(\epsilon-\epsilon_{c}\right) L^{1 / \nu}$. Tuning $\beta$ and $\nu$ to collapse the various system sizes near the critical point, we obtain $\beta_{\mathrm{ED}} \approx 0.4 \pm$ 0.1 and $\nu_{\mathrm{ED}} \approx 1.3 \pm 0.1$ (averaged across all interaction strengths $J_{z}$ ) [25]. These fits are consistent with the exact analytic expression for $\beta$. The value of $\nu_{\mathrm{ED}}$ lies between the expected typical and mean values, likely reflecting the limitations of our small system sizes for capturing rare fluctuations that give $\nu_{\text {avg }}=2$ in macroscopic systems.

Experimental realization.-We now propose a simple experimental blueprint for the implementation of a discrete time crystal in a one dimensional array of trapped ions [40-42]. In such systems, the spin degree of freedom can be formed from two internal electronic states within each ion; an effective transverse field, $H_{T}=\Omega \sum_{i} \sigma_{i}^{x}$, can then be realized via resonant microwave radiation between these electronic states $[41,42]$. Coulomb repulsion between the ions stabilizes a crystalline configuration and interactions between the spins are generated via off resonant laser fields that couple each spin with either longitudinal or transverse phonon modes [43]. This produces long-range Ising-type interactions, $H_{\text {int }}=\sum_{i j} J_{i j} / r_{i j}^{\alpha} \sigma_{i}^{z} \sigma_{j}^{z}$, between the spins which fall off as a tunable power law, with $0<\alpha<3$ (Fig. 4) $[42,43]$. Finally, a disorder potential can be 


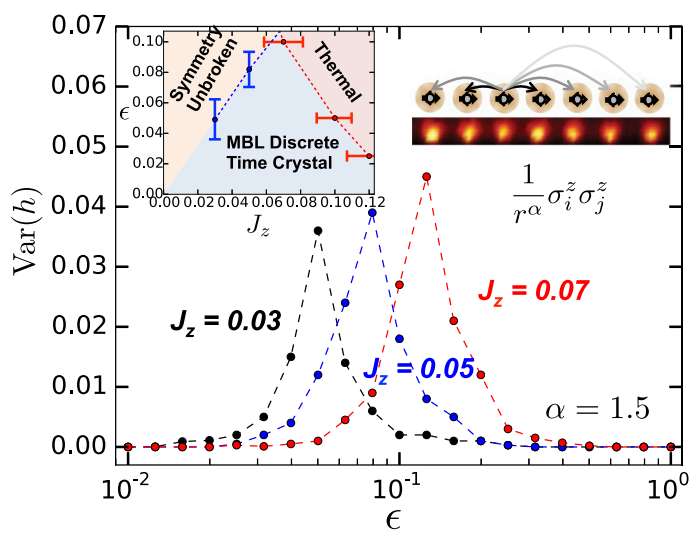

FIG. 4. Trapped ion discrete time crystal-Variance of the $\omega / 2$ Fourier peak magnitude as a function of $\epsilon$ for power-law Ising interactions with $\alpha=1.5$ and $L=10$. Unlike the previous case, here, to mimic the experimental scenario, we begin with the same high-energy-density initial state for all simulations, namely, a polarized product state along $\hat{z}$. The location of the TTSB transition can clearly be seen by the peaking of $\operatorname{Var}(h)$. The top right inset illustrates a schematic of a one dimensional chain of trapped ions interacting via long-range power law interactions. The top left inset depicts the DTC phase diagram for $\alpha=1.5$.

generated via either individual ion addressing or a 1D optical speckle potential that leads to randomized ac Stark shifts $[44,45]$. In combination, these above ingredients enable the direct realization of a power-law generalization of Eq. (1),

$$
U^{\text {ion }}=\left\{\begin{array}{l}
U_{1}^{\text {ion }}=e^{-i \Omega \sum_{i} \sigma_{i}^{\alpha} t_{1}} \\
U_{2}^{\text {ion }}=e^{-i\left(\sum_{i j} J_{i j} / r_{i j}^{\alpha} \sigma_{i}^{z} \sigma_{j}^{z}+B_{i}^{z} \sigma_{i}^{z}\right) t_{2}},
\end{array}\right.
$$

where the nearest neighbor Ising interaction is replaced by $H_{\text {int }}$ and $\left\{t_{1}, t_{2}\right\}$ represent tunable evolution times. We emphasize that our proposed realization can likewise be naturally implemented in ultracold polar molecules $[46,47]$ and Rydberg-dressed neutral atom arrays [48,49], both of which also feature long-range interactions.

This leads to a key question: can the discrete time crystal survive the presence of such long-range interactions [50-52]? To quantitatively probe the effect of the longrange power law and the existence of a DTC phase in trapped ions, we perform a numerical study of $U^{\text {ion }}$ with $\alpha=1.5$ [53]. Diagnosing the MBL transition, one finds that long-range interactions disfavor localization and the MBL transition shifts significantly toward smaller $J_{z}$ [inset Fig. 4, red line] [25]. We note that many-body resonance counting suggests a critical power law, $\alpha_{c}=3 / 2$ in one dimension [52], although this delocalization is expected to emerge only for very large systems, and we do not find evidence of such critical delocalization in our simulations.

Interestingly, within the localized phase, power-law interactions seem to better stabilize the DTC phase [25]. In particular, starting from a fully polarized product state aligned along $\hat{z}$, we again compute the variance of $h$ as a function of $\epsilon$. As illustrated in Fig. 4, the transition as determined from the peaking of $\operatorname{Var}(h)$ is weakly enhanced when compared to the short-range case, leading to a modified phase diagram (Fig. 4, inset). These results suggest that a trapped ion quantum simulator can naturally realize a discrete time crystal phase, even in the presence of long-range interactions. Moreover, within current coherence times [42], one can observe $\sim 10^{2}$ Floquet periods, sufficient to detect both the DTC's subharmonic rigidity and to probe its TTSB transition via $\operatorname{Var}(h)$.

In summary, we have introduced a simple, one dimensional disordered Floquet system that exhibits a robust discrete time crystal phase. We characterize this phase via several diagnostics including the rigidity of the emergent subharmonic frequency to changes or imperfections in the driving. Moreover, we develop a theory of the melting transition from the time crystal into the trivial Floquet paramagnet and utilize this to conjecture a scaling form for the mutual information. Finally, we propose a realization of the discrete time crystal in a 1D array of long-rangeinteracting trapped ions and demonstrate that signatures of both the DTC phase and the TTSB transition can be directly observed with current experimental technologies.

We gratefully acknowledge the insights of and discussions with E. Altman, B. Bauer, P. Hess, D. Huse, V. Khemani, A. Lee, M. Lukin, C. Monroe, C. Nayak, J. Smith, S. Sondhi, C. von Keyserlingk, R. Vasseur, M. Zaletel, and J. Zhang. This work was supported, in part by, the AFOSR MURI Grant No. FA9550-14-1-0035, the Simons Investigator Program, the Gordon and Betty Moore Foundation's EPiQS Initiative through Grant No. GBMF4307, and the Miller Institute for Basic Research in Science.

[1] M. Peskin and D. Schroeder, An introduction to quantum field theory, (1995).

[2] P. M. Chaikin and T. C. Lubensky, Principles of Condensed Matter Physics (Cambridge University Press, Cambridge, England, 2000), Vol. 1.

[3] F. Wilczek, Phys. Rev. Lett. 109, 160401 (2012).

[4] T. Li, Z.-X. Gong, Z.-Q. Yin, H. T. Quan, X. Yin, P. Zhang, L.-M. Duan, and X. Zhang, Phys. Rev. Lett. 109, 163001 (2012).

[5] F. Wilczek, Phys. Rev. Lett. 111, 250402 (2013).

[6] P. Bruno, Phys. Rev. Lett. 111, 070402 (2013).

[7] P. Nozières, Europhys. Lett. 103, 57008 (2013).

[8] G. E. Volovik, JETP Lett. 98, 491 (2013).

[9] H. Watanabe and M. Oshikawa, Phys. Rev. Lett. 114, 251603 (2015)

[10] V. Khemani, A. Lazarides, R. Moessner, and S. L. Sondhi, Phys. Rev. Lett. 116, 250401 (2016).

[11] D. V. Else, B. Bauer, and C. Nayak, Phys. Rev. Lett. 117, 090402 (2016).

[12] C. von Keyserlingk and S. Sondhi, Phys. Rev. B 93, 245146 (2016). 
[13] C. von Keyserlingk, V. Khemani, and S. Sondhi, Phys. Rev. B 94, 085112 (2016).

[14] P. Ponte, Z. Papić, F. Huveneers, and D. A. Abanin, Phys. Rev. Lett. 114, 140401 (2015).

[15] P. Ponte, A. Chandran, Z. Papić, and D. A. Abanin, Ann. Phys. (Amsterdam) 353, 196 (2015).

[16] A. Lazarides, A. Das, and R. Moessner, Phys. Rev. Lett. 115, 030402 (2015).

[17] D. A. Abanin, W. De Roeck, and F. Huveneers, Ann. Phys. (Amsterdam) 372, 1 (2016).

[18] L. D'Alessio and M. Rigol, Phys. Rev. X 4, 041048 (2014).

[19] A. Lazarides, A. Das, and R. Moessner, Phys. Rev. E 90, 012110 (2014).

[20] D. V. Else, B. Bauer, and C. Nayak, arXiv:1607.05277.

[21] K. Sacha, Phys. Rev. A 91, 033617 (2015).

[22] We note that TTSB can also emerge in the large-N limit of certain driven $O(N)$ models, but that finite $N$ always leads to eventual thermalization [23].

[23] A. Chandran and S. L. Sondhi, Phys. Rev. B 93, 174305 (2016).

[24] We note that this phase is related to the $\pi$ spin glass phase $[10,13]$ and is referred to as a Floquet time crystal in [11].

[25] See Supplemental Material at http://link.aps.org/ supplemental/10.1103/PhysRevLett.118.030401 for derivations and additional numerics.

[26] A. T. Winfree, J. Theor. Biol. 16, 15 (1967).

[27] L. Balents and M. P. Fisher, Phys. Rev. Lett. 75, 4270 (1995).

[28] In Fig. 1(b), for $J_{z}=0, \epsilon \neq 0$, there are two peaks in the disorder averaged Fourier spectra. We compute the splitting, $\delta$, between these peaks and plot $\omega / 2+\delta / 2$ at $+\epsilon$ and $\omega / 2-\delta / 2$ at $-\epsilon$.

[29] We work at maximal disorder $W=2 \pi$; unlike equilibrium systems, the periodicity of the Floquet unitary limits the strength of the disorder potential.

[30] A. Pal and D. A. Huse, Phys. Rev. B 82, 174411 (2010).

[31] Recently, using the arguments of [32], the stability of the random Ising critical point against thermalization even in the static MBL setting at strong disorder has been questioned [33]. While this is an important question of principle that remains unsettled, if the mechanism proposed in [32] indeed occurs, then our analytic scaling predictions still provide an accurate description up to exponentially (in disorder strength) large system sizes and superexponentially long time scales. Hence, the mechanism proposed in [32] is unlikely to be a practical limiting factor of observing these effects in either numerics or experiments. Therefore, for our purposes of establishing the boundaries of a phase with TTSB, we follow [34-38] and compare properties of the observed transition with those of the random Ising universality class.
[32] W. De Roeck and F. Huveneers, arXiv:1608.01815.

[33] V. Khemani and D. Huse (private communication).

[34] D. S. Fisher, Phys. Rev. Lett. 69, 534 (1992).

[35] R. Vosk and E. Altman, Phys. Rev. Lett. 112, 217204 (2014).

[36] D. Pekker, G. Refael, E. Altman, E. Demler, and V. Oganesyan, Phys. Rev. X 4, 011052 (2014).

[37] R. Vasseur, A. C. Potter, and S. Parameswaran, Phys. Rev. Lett. 114, 217201 (2015).

[38] Y.-Z. You, X.-L. Qi, and C. Xu, Phys. Rev. B 93, 104205 (2016).

[39] M. Serbyn, Z. Papić, and D. A. Abanin, Phys. Rev. Lett. 111, 127201 (2013).

[40] K. Kim, M.-S. Chang, S. Korenblit, R. Islam, E. Edwards, J. Freericks, G.-D. Lin, L.-M. Duan, and C. Monroe, Nature (London) 465, 590 (2010).

[41] R. Blatt and C. Roos, Nat. Phys. 8, 277 (2012).

[42] J. Smith, A. Lee, P. Richerme, B. Neyenhuis, P. W. Hess, P. Hauke, M. Heyl, D. A. Huse, and C. Monroe, Nat. Phys. 12, 907 (2016).

[43] S. Korenblit, D. Kafri, W. C. Campbell, R. Islam, E. E. Edwards, Z.-X. Gong, G.-D. Lin, L. Duan, J. Kim, K. Kim et al., New J. Phys. 14, 095024 (2012).

[44] M. White, M. Pasienski, D. McKay, S. Zhou, D. Ceperley, and B. DeMarco, Phys. Rev. Lett. 102, 055301 (2009).

[45] S. Kondov, W. McGehee, W. Xu, and B. DeMarco, Phys. Rev. Lett. 114, 083002 (2015).

[46] B. Yan, S. A. Moses, B. Gadway, J. P. Covey, K. R. Hazzard, A. M. Rey, D. S. Jin, and J. Ye, Nature (London) 501, 521 (2013).

[47] K. R. Hazzard, B. Gadway, M. Foss-Feig, B. Yan, S. A. Moses, J. P. Covey, N. Y. Yao, M. D. Lukin, J. Ye, D. S. Jin et al., Phys. Rev. Lett. 113, 195302 (2014).

[48] J. Zeiher, R. van Bijnen, P. Schauß, S. Hild, J.-y. Choi, T. Pohl, I. Bloch, and C. Gross, arXiv:1602.06313.

[49] M. Endres, H. Bernien, A. Keesling, H. Levine, E. R. Anschuetz, A. Krajenbrink, C. Senko, V. Vuletic, M. Greiner, and M. D. Lukin, arXiv:1607.03044.

[50] A. L. Burin, arXiv:cond-mat/0611387.

[51] N. Y. Yao, C. R. Laumann, S. Gopalakrishnan, M. Knap, M. Mueller, E. A. Demler, and M. D. Lukin, Phys. Rev. Lett. 113, 243002 (2014).

[52] A. L. Burin, Phys. Rev. B 92, 104428 (2015).

[53] To compare with the original model, we set $t_{1}=t_{2}=1$, $\Omega=g-\epsilon$, nearest neighbor $J_{i j}=J_{z}$, and $B_{i}^{z} \in[0,2 \pi]$. Inhomogeneities in the spacing of the experimental ion crystal [42] lead to slight modifications in the power law and to this end, we use a coupling matrix that accounts for these in inhomogeneities. 